14. Pre-symptomatic detection of cytoplasmic TDP-43 accumulation using tissue-engineered skin model derived from C9ORF72-FALS patients

B. Paré ${ }^{1,2}$, L. Touzel-Deschênes ${ }^{2}$, R. Lamontagne ${ }^{2}$, M-S. Lamarre ${ }^{2}$, F-D. Scott ${ }^{1,2}$, C. Gaudet ${ }^{1,2}$, H.T. Khuong ${ }^{1,3}$, P. Dion ${ }^{4}$, S. Saikali ${ }^{5}$, J-P. Bouchard ${ }^{3}$, P.V. Gould ${ }^{5}$, G. Rouleau ${ }^{4}$, N. Dupré ${ }^{3}$, F. Berthod ${ }^{1,2}$, F. Gros-Louis ${ }^{1,2}$

${ }^{1}$ Department of Surgery, Faculty of Medicine, Laval University, Quebec City, Canada; ${ }^{2} \mathrm{CHU}$ de Quebec research center, LOEXHôpital de l'Enfant-Jésus, Quebec City, Canada; ${ }^{3}$ ALS clinic, Department of neurological sciences, CHU de Québec and Faculty of Medicine, Laval University, Quebec City, Canada; ${ }^{4}$ Montreal Neurological Institute and Hospital, McGill University, Montreal, Canada; ${ }^{5}$ Department of Medical Biology, section of neuropathology, CHU de Québec, Hôpital de l'Enfant- Jésus, Quebec City, Canada

doi:10.1017/cjn.2015.262

Amyotrophic lateral sclerosis (ALS) is an adult-onset disease characterized by the selective degeneration of motor neurons in the brain and spinal cord resulting in progressive paralysis and death. Current diagnosis of ALS is based on clinical assessment of related symptoms, which appear only late in the disease course after degeneration of a significant number of motor neurons. As a result, the identification and development of disease-modifying therapies is difficult, making ALS an incurable disease. Novel strategies for early diagnosis of ALS, to monitor disease progression and to assess response to existing and future treatments are urgently needed.

Many neurological disorders, including ALS, are accompanied by skin changes that often precede the onset of neurological symptoms. We have developed a unique ALS tissue-engineered skin model (ALS-TES), derived from the cells of ALS patients, in order to study the earliest stages of ALS-related skin pathology. For each participant, two skin biopsies were collected using a 6-mm diameter punch biopsy. Tissue-engineered skin was then generated from isolated keratinocytes and fibroblasts, and examined by routine histochemistry and immunohistochemistry, as well as by confocal microscopy. The ALS-TES model presents a number of striking features including altered epidermal differentiation, abnormal dermo-epidermal junction, delamination, keratinocyte infiltration, collagen disorganization and cytoplasmic TDP-43 inclusions, which are not seen in skin models derived from healthy subjects. The same abnormal skin model changes were detected skin models derived from the cells of pre- symptomatic C9orf72-linked ALS patients carrying the GGGGCC DNA repeat expansion. Consequently, our ALS-TES skin model could represent a renewable source of human tissue to better understand the physiopathological mechanisms underlying this disease, including cytoplasmic TDP43 accumulation, and lead to better tools for early diagnosis and disease monitoring.

\section{Titles of Diagnostic Case Presentations}

\section{Myxopapillary Ependymoma, multifocal}

P.W. Schutz ${ }^{1}$, G.R.W. Moore ${ }^{1}$, R. Akagami ${ }^{2}$, I.R. Mackenzie ${ }^{1}$

${ }^{1}$ Department of Pathology ${ }^{2}$ Division of Neurosurgery Vancouver General Hospital, University of British Columbia, Vancouver, BC, Canada

\section{Phosphaturic mesenchymal tumor}

P Diamandis $^{1}$, MD Cusimano $^{2}$, K Kovacs $^{1}$, J Karamchandani $^{3}$

${ }^{1}$ Department of Laboratory Medicine, Division of Pathology, St Michael's Hospital, University of Toronto, Toronto, ON, Canada. ${ }^{2}$ Division of Neurosurgery, Department of Surgery, St. Michael's Hospital, University of Toronto, Toronto, ON, Canada. ${ }^{3}$ McGill University, Montreal Neurological Institute and Hospital, Montreal, Quebec, Canada.

\section{Secondary Gliosarcoma.with Epithelial and Pseudoepithelial Differentiation}

\section{Claire I. Coiré ${ }^{1}$, And Warren P. Mason ${ }^{2}$}

${ }^{1}$ Department of Pathology, Trillium Health Partners, Mississauga and ${ }^{2}$ Department of Medical Oncology, Pencer Brain Tumor Centre, Princess Margaret Cancer Centre, University of Toronto.

\section{Lymphomatosis cerebri}

$$
\text { J.P. Rossiter }{ }^{1} \text {, A. Adeyinka }{ }^{1} \text { and J.G. Boyd }{ }^{2}
$$

Department of Pathology and Molecular Medicine ${ }^{1}$ and Division of Neurology 2, Queen's University and Kingston General Hospital, Kingston, Ontario

\section{Myopathy with Hexagonally Cross-Linked Crystalloid} Inclusions

M. Alturkustani ${ }^{1,2}, F$. AlSufiani ${ }^{l}, L_{-C}$. Ang ${ }^{1}$

${ }^{1}$ Division of Neuropathology, London Health Sciences Centre; University of Western, London, Canada; ${ }^{2}$ Department of Pathology, King Abdulaziz University and Hospital, Jeddah, Saudi Arabia

\section{Bilateral brain stem tegmental calcification}

N. Sinha ${ }^{1}$, R. Fraser ${ }^{2}$, CJ. Fallet-Bianco ${ }^{3} \&$ A. Oviedo ${ }^{2}$

${ }^{1}$ Division of Anatomical Pathology, Queen Elizabeth II Health Sciences Centre and Dalhousie University, Halifax, Nova Scotia, Canada; ${ }^{2}$ Department of Pathology \& Laboratory Medicine, IWK Health Centre, Halifax, Canada ${ }^{3}$ Department of Pathology and Neuropathology, Hôpital Sainte-Justine, Montreal, Canada 